\title{
Cation- $\pi$ Interactions of a Thiocarbonyl Group and a Carbonyl Group With a Pyridinium Nucleus
}

\author{
Shinji Yamada*, Tomoko Misono and Seiji Tsuzuki ${ }^{\ddagger}$
}

\section{Supporting Information}

Table S1. The HF/6-311G** level optimized geometry and the calculated energy of 1 (Figure 7).

Table S2. The HF/6-311G** level optimized geometry and the calculated energy of 2a (Figure 7).

Table S3. The geometry and the calculated energy of the model complex $\left(\mathrm{CH}_{2} \mathbf{S}-\mathbf{C}_{5} \mathbf{H}_{5} \mathbf{N M e}^{+}\right)$at the potential energy minimum shown in Figure 8.

Table S4. The geometry and the calculated energy of the model complex $\left(\mathrm{CH}_{2} \mathbf{O}-\mathbf{C}_{5} \mathbf{H}_{5} \mathbf{N M e}^{+}\right)$at the potential energy minimum shown in Figure 8.

Table S5. The geometry of the model complex $\left(\mathrm{CH}_{2} \mathbf{S}-\mathbf{C}_{5} \mathbf{H}_{5} \mathbf{N M e}^{+}\right)$used for the calculations of the Ees and Eind (Figure 9).

Table S6. The geometry of the model complex $\left(\mathrm{CH}_{2} \mathbf{O}-\mathbf{C}_{5} \mathbf{H}_{5} \mathbf{N M e}^{+}\right)$used for the calculations of the Ees and Eind (Figure 9). 


\section{Table S1}

The HF/6-311G** level optimized geometry and the calculated energy of 1 (Figure 7).

\begin{tabular}{|c|c|c|c|}
\hline 16 & 0.67957 & -1.78852 & -1.16994 \\
\hline 16 & 3.09068 & -0.80861 & 0.24293 \\
\hline 8 & -0.27505 & 2.21328 & -0.93903 \\
\hline 7 & 0.97531 & 0.61911 & 0.02879 \\
\hline 7 & -3.86136 & 0.24886 & -0.40352 \\
\hline 6 & -0.27266 & 1.16331 & -0.39503 \\
\hline 6 & -2.68703 & 0.76354 & -0.69938 \\
\hline 1 & -2.65041 & 1.44876 & -1.52604 \\
\hline 6 & -1.52033 & 0.45873 & -0.00859 \\
\hline 6 & -1.60334 & -0.41920 & 1.06018 \\
\hline 1 & -0.73025 & -0.68033 & 1.62764 \\
\hline 6 & -2.83424 & -0.94925 & 1.38610 \\
\hline 1 & -2.94815 & -1.63061 & 2.20790 \\
\hline 6 & -3.92686 & -0.58664 & 0.61703 \\
\hline 1 & -4.90095 & -0.98825 & 0.83751 \\
\hline 6 & 1.43656 & -0.61416 & -0.31529 \\
\hline 6 & 2.03241 & 1.59293 & 0.33762 \\
\hline 1 & 1.62051 & 2.39075 & 0.93797 \\
\hline 1 & 2.41992 & 2.01527 & -0.58166 \\
\hline 6 & 3.09893 & 0.80416 & 1.07662 \\
\hline 1 & 2.85272 & 0.67721 & 2.12228 \\
\hline 1 & 4.07473 & 1.25882 & 0.98641 \\
\hline
\end{tabular}




\section{Table S2}

The HF/6-311G** level optimized geometry and the calculated energy of 2a (Figure 7).

\begin{tabular}{|c|c|c|c|}
\hline 8 & -1.97300 & -2.01195 & -1.40327 \\
\hline 6 & -1.93642 & -1.17042 & -0.57529 \\
\hline 16 & -1.89690 & 1.98063 & -0.14260 \\
\hline 16 & -4.77800 & 1.42010 & 0.19936 \\
\hline 7 & -3.08298 & -0.45691 & -0.21817 \\
\hline 6 & -3.13363 & 0.90835 & -0.05585 \\
\hline 6 & -4.38024 & -1.06233 & -0.58446 \\
\hline 6 & -5.41689 & -0.28141 & 0.20293 \\
\hline 1 & -4.37007 & -2.11104 & -0.32949 \\
\hline 1 & -4.53421 & -0.96215 & -1.65144 \\
\hline 1 & -6.38575 & -0.30433 & -0.27291 \\
\hline 1 & -5.50473 & -0.63527 & 1.22075 \\
\hline 7 & 1.68634 & -1.11738 & 0.15921 \\
\hline 6 & 0.50961 & -1.22876 & -0.45328 \\
\hline 6 & -0.66803 & -0.95127 & 0.19871 \\
\hline 6 & -0.61568 & -0.59519 & 1.53593 \\
\hline 6 & 0.61374 & -0.51578 & 2.17130 \\
\hline 6 & 1.74944 & -0.76686 & 1.45021 \\
\hline 6 & 2.95099 & -1.43262 & -0.59067 \\
\hline 6 & 4.00992 & -0.37253 & -0.42527 \\
\hline 6 & 3.82546 & 0.89698 & -0.96470 \\
\hline 6 & 4.80672 & 1.85821 & -0.82746 \\
\hline 6 & 5.98505 & 1.55752 & -0.15745 \\
\hline 6 & 6.17767 & 0.29589 & 0.37228 \\
\hline 6 & 5.18968 & -0.66890 & 0.23905 \\
\hline
\end{tabular}




$\begin{array}{rrrr}1 & 0.51395 & -1.53549 & -1.47990 \\ 1 & -1.51689 & -0.38459 & 2.07901 \\ 1 & 0.69200 & -0.24540 & 3.20566 \\ 1 & 2.73007 & -0.69019 & 1.87546 \\ 1 & 2.66008 & -1.54149 & -1.62461 \\ 1 & 3.29551 & -2.39281 & -0.23310 \\ 1 & 2.92038 & 1.13602 & -1.49694 \\ 1 & 4.66052 & 2.83600 & -1.24800 \\ 1 & 6.75001 & 2.30540 & -0.05693 \\ 1 & 7.09206 & 0.05708 & 0.88327 \\ 1 & 5.35158 & -1.65311 & 0.64398 \\ & & & \\ \text { E(RHF) }= & -1593.43357541 & \text { A.U. }\end{array}$




\section{Table S3}

The geometry and the calculated energy of the model complex $\left(\mathbf{C H}_{2} \mathbf{S}-\mathbf{C}_{5} \mathbf{H}_{5} \mathbf{N M e}^{+}\right)$at the potential energy minimum shown in Figure 8.

\begin{tabular}{|c|c|c|c|}
\hline \multicolumn{4}{|l|}{$\mathrm{R}=4.0 \mathrm{~A}$} \\
\hline 7 & -1.279834 & 1.655106 & .000000 \\
\hline 6 & -1.279834 & 3.139006 & .000000 \\
\hline 6 & -1.279834 & .993217 & 1.180162 \\
\hline 6 & -1.279834 & .993217 & -1.180162 \\
\hline 6 & -1.279834 & -.396292 & 1.203201 \\
\hline 6 & -1.279834 & -.396292 & -1.203201 \\
\hline 6 & -1.279834 & -1.106294 & .000000 \\
\hline 1 & -1.802004 & 3.485174 & -.890256 \\
\hline 1 & -1.802004 & 3.485174 & .890256 \\
\hline 1 & -.247969 & 3.493009 & .000000 \\
\hline 1 & -1.279834 & 1.602397 & 2.076677 \\
\hline 1 & -1.279834 & 1.602397 & -2.076677 \\
\hline 1 & -1.279834 & -.904064 & 2.161145 \\
\hline 1 & -1.279834 & -.904064 & -2.161145 \\
\hline 1 & -1.279834 & -2.191494 & .000000 \\
\hline 16 & 2.720166 & -1.106294 & .000000 \\
\hline 6 & 2.720166 & -2.719849 & .000000 \\
\hline 1 & 1.794785 & -3.296935 & .000000 \\
\hline 1 & 3.645548 & -3.296935 & .000000 \\
\hline $\mathrm{E}(\mathrm{RHF})=$ & -722.710459119 & A.U. & \\
\hline EUMP2 = & -.72398403621 & $93 \mathrm{D}+03$ & \\
\hline
\end{tabular}




\section{Table S4}

The geometry and the calculated energy of the model complex $\left(\mathrm{CH}_{2} \mathbf{O}-\mathbf{C}_{5} \mathbf{H}_{5} \mathbf{N M e}^{+}\right)$at the potential energy minimum shown in Figure 8.

\begin{tabular}{|c|c|c|c|}
\hline \multicolumn{4}{|l|}{$\mathrm{R}=3.4 \mathrm{~A}$} \\
\hline 7 & -.811754 & 1.475268 & .000000 \\
\hline 6 & -.811754 & 2.959168 & .000000 \\
\hline 6 & -.811754 & .813379 & 1.180162 \\
\hline 6 & -.811754 & .813379 & -1.180162 \\
\hline 6 & -.811754 & -.576130 & 1.203201 \\
\hline 6 & -.811754 & -.576130 & -1.203201 \\
\hline 6 & -.811754 & -1.286132 & .000000 \\
\hline 1 & -1.333924 & 3.305335 & -.890256 \\
\hline 1 & -1.333924 & 3.305335 & .890256 \\
\hline 1 & .220111 & 3.313171 & .000000 \\
\hline 1 & -.811754 & 1.422559 & 2.076677 \\
\hline 1 & -.811754 & 1.422559 & -2.076677 \\
\hline 1 & -.811754 & -1.083902 & 2.161145 \\
\hline 1 & -.811754 & -1.083902 & -2.161145 \\
\hline 1 & -.811754 & -2.371332 & .000000 \\
\hline 8 & 2.588246 & -1.286132 & .000000 \\
\hline 6 & 2.588246 & -2.496967 & .000000 \\
\hline 1 & 1.651991 & -3.085529 & .000000 \\
\hline 1 & 3.524501 & -3.085529 & .000000 \\
\hline $\mathrm{E}(\mathrm{RHF})=$ & -400.068639429 & A.U. & \\
\hline EUMP2 = & -.40140730959 & $47 \mathrm{D}+03$ & \\
\hline
\end{tabular}




\section{Table S5}

The geometry of the model complex $\left(\mathrm{CH}_{2} \mathbf{S}-\mathbf{C}_{5} \mathbf{H}_{5} \mathbf{N M e}^{+}\right)$used for the calculations of the Ees and Eind (Figure 9).

\begin{tabular}{|c|c|c|c|}
\hline 7 & -2.05140 & 4.14040 & 11.12330 \\
\hline 6 & -1.74940 & 4.67430 & 12.30740 \\
\hline 6 & -0.46330 & 5.09200 & 12.57820 \\
\hline 6 & 0.52980 & 4.85360 & 11.65100 \\
\hline 6 & 0.20270 & 4.22600 & 10.45280 \\
\hline 6 & -1.10060 & 3.89560 & 10.19200 \\
\hline 6 & -3.49490 & 3.90020 & 10.77710 \\
\hline 1 & -2.55763 & 4.81308 & 12.99806 \\
\hline 1 & -0.25206 & 5.55713 & 13.52136 \\
\hline 1 & 1.54392 & 5.14728 & 11.84835 \\
\hline 1 & 0.95257 & 4.01736 & 9.71446 \\
\hline 1 & -1.42466 & 3.45081 & 9.27253 \\
\hline 1 & -4.03424 & 3.67873 & 11.68438 \\
\hline 1 & -3.88870 & 4.79016 & 10.30780 \\
\hline 1 & -3.55392 & 3.05813 & 10.10570 \\
\hline 16 & -0.83600 & 7.99480 & 11.71690 \\
\hline 6 & 0.26380 & 7.95370 & 12.92200 \\
\hline 1 & 0.31811 & 7.12527 & 13.61025 \\
\hline 1 & 0.97198 & 8.75358 & 13.06905 \\
\hline
\end{tabular}




\section{Table S6}

The geometry of the model complex $\left(\mathrm{CH}_{2} \mathbf{O}-\mathbf{C}_{5} \mathbf{H}_{5} \mathbf{N M e}^{+}\right)$used for the calculations of the Ees and Eind (Figure 9).

\begin{tabular}{|c|c|c|c|}
\hline 7 & 8.88760 & 0.54410 & 6.53710 \\
\hline 6 & 8.41990 & 1.52410 & 7.35010 \\
\hline 6 & 7.27600 & 2.19270 & 6.96160 \\
\hline 6 & 6.67220 & 1.93720 & 5.76130 \\
\hline 6 & 7.18610 & 0.94460 & 4.96620 \\
\hline 6 & 8.27590 & 0.21130 & 5.37850 \\
\hline 6 & 10.14380 & -0.17920 & 6.91680 \\
\hline 1 & 8.95586 & 1.71894 & 8.25640 \\
\hline 1 & 6.89142 & 2.95337 & 7.61483 \\
\hline 1 & 5.80682 & 2.49391 & 5.45465 \\
\hline 1 & 6.73029 & 0.69701 & 4.02635 \\
\hline 1 & 8.70312 & -0.59153 & 4.81209 \\
\hline 1 & 10.26369 & -0.12790 & 7.98721 \\
\hline 1 & 10.05426 & -1.21084 & 6.61395 \\
\hline 1 & 10.98158 & 0.29017 & 6.42103 \\
\hline 8 & 6.72260 & 0.99730 & 9.54020 \\
\hline 6 & 6.71790 & 2.14530 & 9.89180 \\
\hline 1 & 6.77888 & 2.96611 & 9.17381 \\
\hline 1 & 6.65130 & 2.42279 & 10.94609 \\
\hline
\end{tabular}

